\title{
Deciphering the binding mechanism of Dexamethasone against SARS-CoV-2 Main Protease: Computational molecular modelling approach
}

\author{
Shafi Ullah Khan ${ }^{1 *}$, Thet-Thet Htar ${ }^{1 *}$ \\ ${ }^{1}$ School of Pharmacy, Monash University Malaysia, Jalan Lagoon Selatan, Bandar Sunway, \\ 47500 Subang Jaya, Selangor, Malaysia \\ *Correspondence: thet.thet.htar@monash.edu (T.T.H), Tel: +603 5514 4912; \\ shafi.khan1@monah.edu (S.U.K)
}

\begin{abstract}
At present, there are no proven agents for the treatment of 2019 coronavirus disease (COVID-19). The available evidence has not allowed guidelines to clearly recommend any drugs outside the context of clinical trials. One of the most important SARS-CoV-2 protein targets for therapeutics is the $3 \mathrm{C}$-like protease (main protease, Mpro). Here in this study we utilize the recently published 6W63 crystal structure of Mpro complexed with a non-covalent inhibitor X77. Various docking methods FRED, HYBRID, CDOCKER and LEADFINDER tools were benchmark to optimally re-dock the co-crystal ligand within the active site of SARS-COV-2 Mpro. This study was restricted to molecular docking without validation by molecular dynamics simulations. CDOCKER was found to depict the exact binding of cocrystal ligand having lowest RMSD of less than 2 A. Interactions with the SARS-COV-2 Mpro may play a key role in fighting against viruses. Dexamethasone was found to bind with a high affinity to the same sites of the SARS-COV-2 Mpro than the Remdesivir.

Dexamethasone was forming six hydrogen bonds compared to the three hydrogen bonds formed by Remdesivir within the active site of SARS-COV-2 Mpro. LEU141, GLY143, HIS163, GLU166, GLN192 were the key amino acid residue of SAR-COV-2 Mpro involved in stabilizing the complex between Dexamethasone and SARS-COV-2 Mpro. The results suggest the effectiveness of Dexamethasone as potent drugs against SARS-CoV-2 since it bind tightly to its Mpro. In addition, the results also suggest that dexamethasone as top antiviral treatments option than the Remdesivir with high potential to fight the SARS-CoV-2
\end{abstract}

Keywords: SARS-COV-2, Dexamethasone, Main Protease, MPro, Molecular Docking, COVID-19, Treatment, Insilico

\section{Introduction}

The current emergency due to the worldwide spread of the COVID-19 caused by the new severe acute respiratory syndrome coronavirus 2 (SARS-CoV-2) is a great concern for global public health [1, 2] . By 17 June 2020, SARS-CoV-2 has caused more than 8061550 cases and also caused death of 440290 [3]. The recently emerged 2019 Novel Coronavirus (SARSCoV-2) and associated COVID-19 disease also cause serious or even fatal respiratory tract infection and yet no approved therapeutics or effective treatment is currently available to effectively combat the outbreak [4]. This urgent situation is pressing the world to respond with the development of novel vaccine or a small molecule therapeutics for SARS-CoV-2 [5, 6].

There is no approved antiviral drug for treatment of COVID-19 [5]. On June 16 2020, the initial clinical trial results from the United Kingdom (UK) shows dexamethasone, a corticosteroid, 
can be lifesaving for patients who are critically ill with COVID-19 [7, 8]. For patients on ventilators, the treatment was shown to reduce mortality by about one third, and for patients requiring only oxygen, mortality was cut by about one fifth, according to preliminary findings shared with WHO [9]. The benefit was only seen in patients seriously ill with COVID-19, and was not observed in patients with milder disease [8]. Dexamethasone is a synthetic glucocorticoid with potent anti-inflammatory effects that is widely used to treat inflammatory diseases [10]. Dexamethasone has been used since the 1960s to reduce inflammation in a range of conditions, including inflammatory disorders and certain cancers [11, 12]. It has been listed on the WHO Model List of Essential Medicines since 1977 in multiple formulations, and is currently off-patent and affordably available in most countries [13, 14].

Various clinicians and researchers are engaged in investigating and developing antivirals using different strategies combining experimental and in-silico approaches with the goal of identifying novel, selective and potent therapeutic agents. [6, 15-18] Though Dexamethasone is used for more than half century year for various therapeutic option but to date no insight into the binding mechanism of Dexamethasone with SARS-CoV-2 main protease (Mpro) has been reported [19].

An attractive drug target among coronaviruses is the main protease (Mpro, 3CLpro) as shown in figure 1, due to its essential role in processing the polyproteins that are translated from the viral RNA [20]. Mpro/3CLpro is active in its dimer state but till now there is no crystal structure available for the dimer form. Its monomer inhabits the 306 amino acids including 3 domains, folded into helices and b-strands.[6] The SARS-CoV 3CLpro has an important function and is considered an active target for antiviral drugs. Many 3CLpro inhibitors have been reported over the past decade and a variety of inhibitors have been found through screening and structurebased design [21]. By the end of April 2020, additional Mpro crystal structures had been released on the Protein Data Bank website, but only one of them (PDB: 6W63) contained X77, as a non-covalent inhibitor of Mpro [22]. Within the scientific community, most of the current research efforts focus on finding potential SARS-CoV-2 Mpro covalent inhibitors while the possibilities to identify non-covalent inhibitors remain less investigated [23] The present study focused on the SAR-COV-2 Mpro as potential target proteins for Dexamethasone in COVID19 treatment .

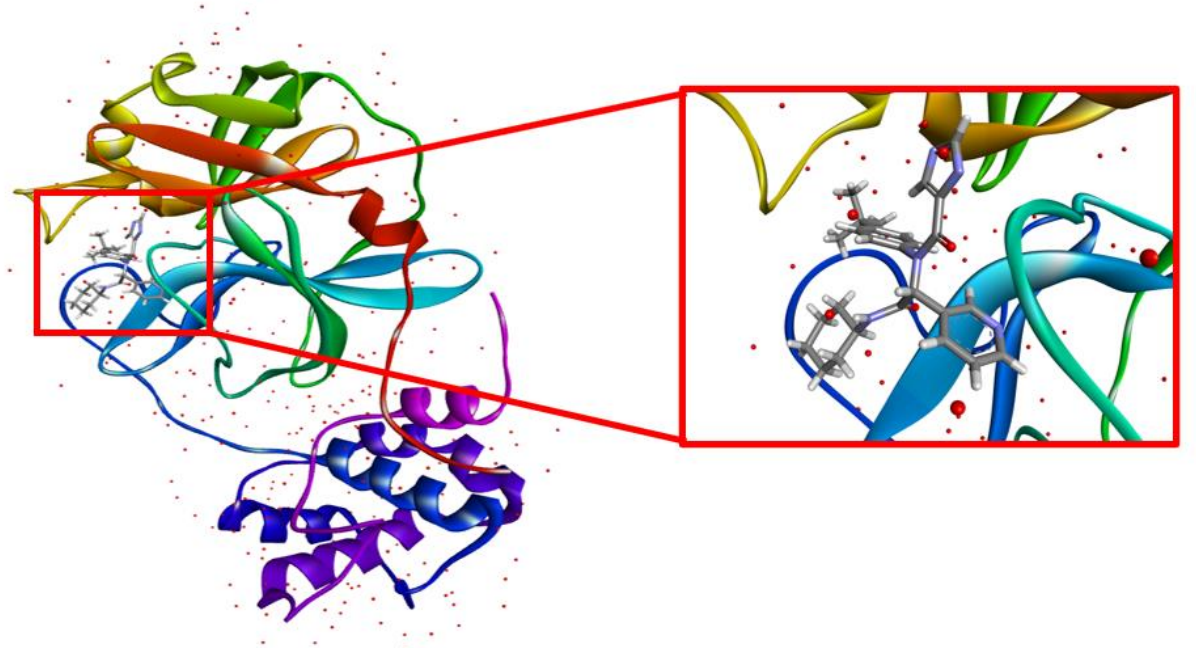


Figure 1: Representation of three dimensional cyrstal structure of SARS-COV-2 Mpro in complex with co-crystal Ligand X77 as a non-covalent inhibitor

To investigate the binding mode of Dexamethasone within SARS-COV2 main protease, we examined the binding interaction of Dexamethasone in comparison with the Remdesivir using multiple computational methods. In first step, we benchmark few molecular docking methods for selecting the best molecular docking approach. With this aim first we re-docked crystal ligand within the active site of SARS-COV-2 Mpro and after getting optimized protocol we used that for investigating the binding free energy and interacting residue involve in making the stable Dexamethasone-SARS-COV-2 Mpro complex. The findings from this study may provide first insight into the computational binding mechanism of dexamethasone with SASCOV-2 Mpro which can be use further in the subsequent exploration as anti-COVID-19 therapeutics.

\section{Material and Method}

\section{Retrieval and preparation of Protein}

Crystal Structure of the COVID-19 virus main protease Mpro (also known as 3CL protease) in complex with potent broad-spectrum non-covalent inhibitor X77 was downloaded from RCSB using PDB ID 6W63 having resolution: 2.10 A [22] (https://www.rcsb.org/structure/6w63). The co-crystal ligand X77 (N-(4-tert-butylphenyl)-N[(1R)-2-(cyclohexylamino)-2-oxo-1-(pyridin-3-yl)ethyl]-1H-imidazole-4-carboxamide) was used as a control in the optimization of molecular docking protocol. Prior to docking calculation Protein Preparation module of Discovery Studio client v20.1 was used to process and prepare the SARS-COV-2 Mpro.[24] This Wizard allows to properly prepare a protein for molecular docking studies.

\section{Retrieval and preparation of ligands}

In the present study, 3D structure of Remdesivir (ID: P121304016) and Dexamethasone (ID:5743) were retrieved from the PubChem webserver https://pubchem.ncbi.nlm.nih.gov/. Prior to testing the Dexamethasone and Remdesivir against SARS-CoV-2 Mpro, the structures of the both molecules were ensured to be in the optimized active form. Optimization was performed by energy minimization, addition of hydrogen atoms and charges if required Ligand Prep Module in the Discovery Studio client v20 [24] was used for ligand optimization. In case of performing molecular docking by OpenEye Scientific Software tools, OMEGA tool was also used to generate the pre-requisite multiple conformers of each ligands [25].

\section{Molecular Docking}

\section{Validation of molecular docking Protocol}

Redocking of co-crystal ligand X77 within the active site of SARS-COV2-Mpro was carried out to properly optimized the molecular docking methods. Selection of best tool for calculating the exact pose of co-crystal ligand X77 is also essential for molecular docking of dexamethasone and Remdesivir within the SAR-COV-2 Mpro. For proper optimization of molecular docking protocol, in first step, various molecular docking tool having different scoring algorithmic were tested by redocking the co-crystal ligand X77. Various molecular docking modules such as CDOCKER tool of Discovery Studio client, FRED and HYBRID module of OpenEye Scientific Software and BioMolTech LeadFinder Virtual screening (VS) 
and extra precision (XP) were checked for redocking the ligand X77 within SAR-COV-2 Mpro [24-28]. Each of the above mentioned molecular redocking application requirs definition of binding site for calculating molecular docking studies. In all docking provedure binding site was defined upon the X77 co-crystal ligand. "Define Site" option in Discovery Studio Client [24] while pdb2receptor tool in OpenEye Scientific Software was utilized for defining the active site prior to docking in FRED and HYBRID [27]. In case of BioMolTech LeadFinder "Energy Grid Maps" were calculated based on co-crystal ligand X77 as a reference ligand [28]. After selecting binding site of SARS-COV-2 Mpro, redocking of X77 was carryout by using default setting in each Docking Program. To cross-validate the docking calculation, docked poses of X77 were superimposed over co-crystal ligand of SARS-COV-2. RMSD was calculated for each co-crystal and re-docked ligand X77. Based on lowest RMSD between cocrystal and re-docked ligand X77 we selected the best docking tool for subsequent molecular docking and binding interaction studies.

\section{Molecular Docking of dexamethasone and Remdesivir in SARS-COV-2 Mpro}

After the successful optimization of docking protocol, in next step molecular docking of dexamethasone and Remdesivir were carried out within the SARS-COV-2 Mpro. Docking score from FRED and HYBRID tool while binding free energy in the form of delta $G(d G)$ from BioMoLTech LeadFinder VS and XP as well as CDOCKER tool were calculated. Base on the lowest binding energy, best docked poses of Dexamethasone and Remdesivir was selected for the visualization of binding interactions with amino acid residue of SARS-COV-2 Mpro. Visualization of ligand-protein complexes were carried out using Discovery studio visualizer [24].

\section{Results and Discussion}

Schematic diagram of Workflow used in the present study is depicted in figure 2 .

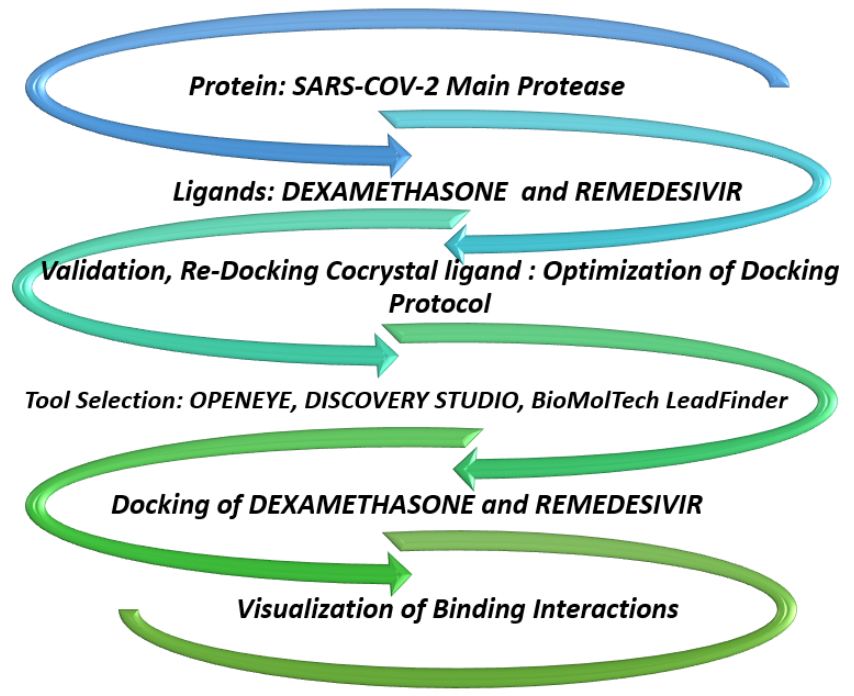

Figure 2: Schematic diagram of Workflow used in the current study

\section{Validation of Docking Protocol}


Proper validation of molecular docking is critical for subsequent use of molecular docking methods. In this study, validation of molecular docking was performed by redocking the cocrystal ligand X77 within the SARS-COV-2 Mpro. Root mean square deviation (RMSD) was calculated for redocked co-crystal and re-docked ligand X77. Detail of calculated RMSD are summarized in table 1 while 3D representation of redocked ligand superimposed on co-crystal ligand X77 are shown in figure 2. Analysis of RMSD calculation from various docking tools revealed that CDOCKER from Discovery (RMSD of 1.0154 $\AA$ ) and LEADFINDER XP (RMSD of $1.0562 \AA$ ) outperform other molecular docking tool.

Table 1: Validation of Re-docking by RMSD profile of redocking co-crystal ligand using different docking Software Protocol

\begin{tabular}{lr}
\hline Name & RMSD (̊) \\
\hline X77_FRED & 7.8174 \\
\hline X77_HYBRID & 6.4435 \\
\hline X77_LEADFINDER VS & 4.3560 \\
\hline X77_LEADFINDER XP & 1.0562 \\
\hline X77_CDOCKER & 1.0154 \\
\hline
\end{tabular}

Redocking of ligand X77 within SARS-COV-2 Mpro using FRED, HYBRID and LEADFINDER VS showed relatively less accurate poses than the poses obtained from the CDOCKER and LEADFINER XP tool. Based on RMSD calculation, we can assumed that CDOCKER and LEADFINDER XP are best depicting docking approach in finding best SARSCOV-2 inhibitor than the other virtual Screening approach. FRED, HYBRID and LEADFINDER VS are virtual screening approaches while LEADFINDE XP and binding energy calculation in CDOCKER tool are relatively computational extensive approach.

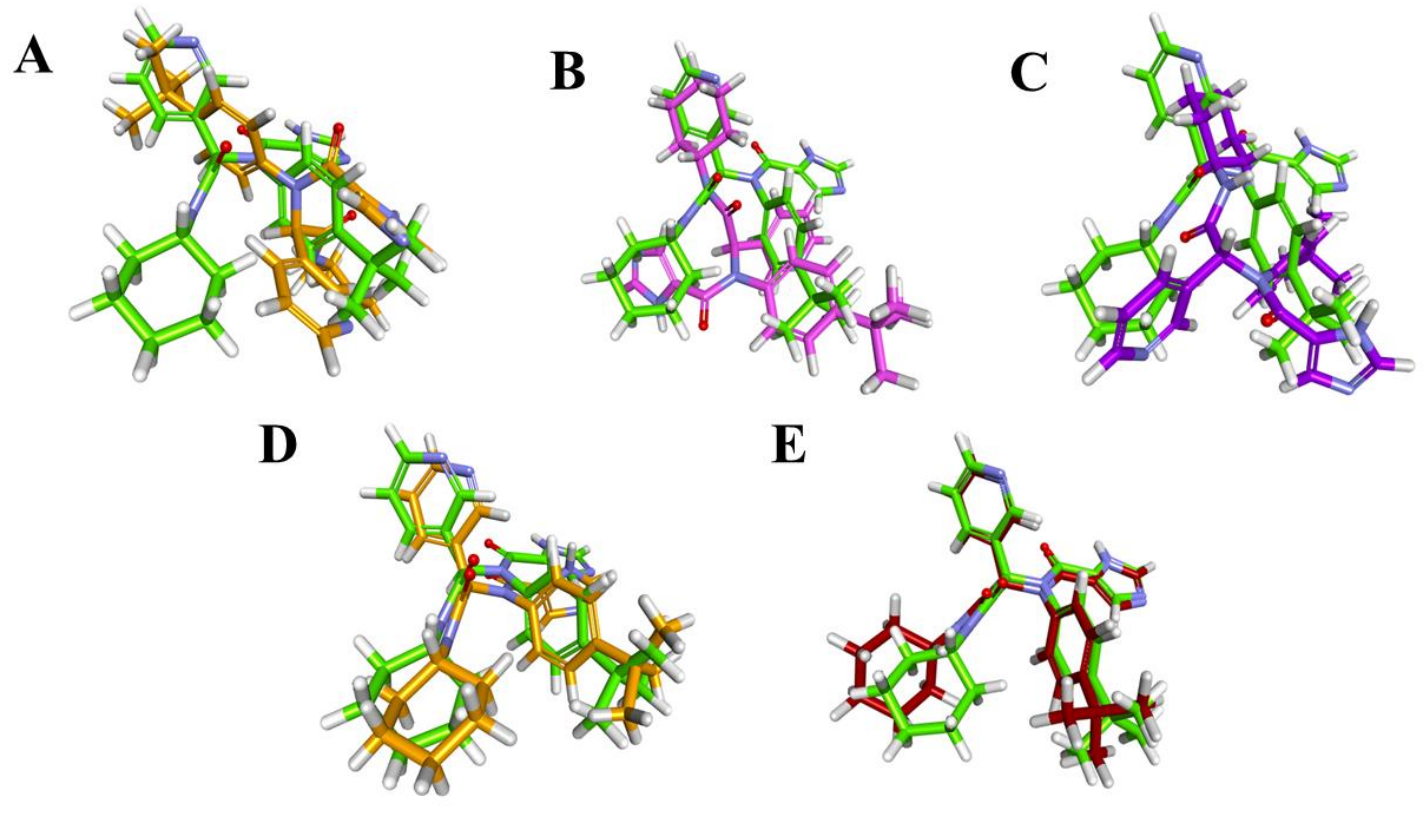


Figure 2. Graphical representation of co-crystal X77 (green coloured) and redocked X77 ligand. A, B, D, D E representing the redocked poses by FRED, HYBRID, LEADFINDER Virtual Screening (VS), LEADFINDER extra precision (XP) and CDOCKER docking respectively.

X77 is the ligand molecule extracted from the crystal structure of SARS-COV-2 Mpro, which was used for defining the binding site in SAR-COV-2. According to the analysis of docking results in Table 2 and Figure 3, the interactions between X77 and binding sites residues are highly consistent with that of Co-crystal ligand. Ligand X77 formed three hydrogen bonds with the amino acid residue of GLY143, HIS163, and GLU166 as shown in figure 2. Additionally MET49, LEU141, HIS41, Leu27 and CyS145 were also involved in forming other hydrophobic interactions with X77 ligand.

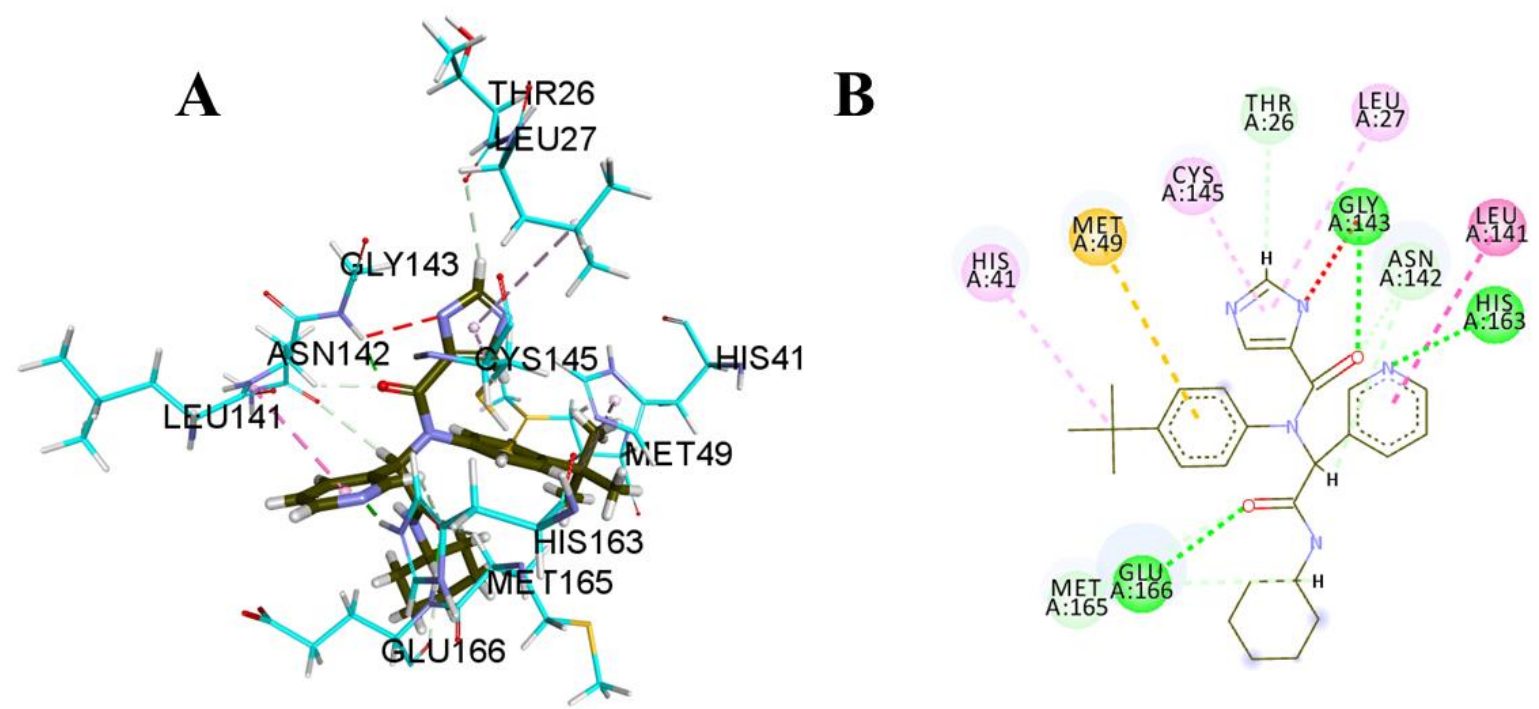

Figure 2. Co-crystal X77 ligand (dark brown colour) which was well stabilised by various amino acid residue (cyan colour) within the active pocket of SARS-CoV-2 Main Protease. Hydrogen bonding are shown in green dotted line, pi-sulfur are shown in golden dotted line while pi-pi T-Shaped interaction are shown in pink dotted line.

Table 2: Detailed Binding Interactions of Redocked ligand X77 within the active site of SARS-COV-2 Mpro.

\begin{tabular}{|l|l|l|}
\hline Type of Bond Hydrogen & From & A:GLY143:HN \\
\hline $\begin{array}{l}\text { Conventional Hydrogen } \\
\text { Bond }\end{array}$ & A:HIS163:HE2 & A:X77401:O01 \\
\hline $\begin{array}{l}\text { Conventional Hydrogen } \\
\text { Bond }\end{array}$ & A:GLU166:HN & A:X77401:N18 \\
\hline $\begin{array}{l}\text { Conventional A:X77401:O13 } \\
\text { Bond }\end{array}$ & A:ASN142:HA & A:X77401:O01 \\
\hline Carbon Hydrogen Bond & A:MET165:HA & A:X77401:O13 \\
\hline Carbon Hydrogen Bond & A:X77401:H041 & A:ASN142:OD1 \\
\hline Carbon Hydrogen Bond & \\
\hline
\end{tabular}




\begin{tabular}{|l|l|l|}
\hline Carbon Hydrogen Bond & A:X77401:H071 & A:GLU166:O \\
\hline Carbon Hydrogen Bond & A:X77401:H331 & A:THR26:O \\
\hline Pi-Sulfur & A:MET49:SD & A:X77401 \\
\hline Amide-Pi Stacked & A:LEU141:C,O;ASN142:N & A:X77401 \\
\hline Pi-Alkyl & A:HIS41 & A:X77401:C24 \\
\hline Pi-Alkyl & A:X77401 & A:LEU27 \\
\hline Pi-Alkyl & A:X77401 & A:CYS145 \\
\hline
\end{tabular}

\section{Molecular Docking of dexamethasone and Remdesivir in SARS-COV-2 Mpro}

To date there is no FDA approved antiviral drug for the treatment of COVID-19. Preliminary report describes the clinical outcomes in a small cohort of patients who were severely ill with Covid-19 and were treated with Remdesivir [29]. On June 17, 2020 WHO welcomes initial clinical trial results from the United Kingdom that show dexamethasone, a corticosteroid, can be lifesaving for patients who are critically ill with COVID-19. According to preliminary findings shared with WHO, for patients on ventilators, the treatment was shown to reduce mortality by about one third, and for patients who only require oxygen, mortality was cut by about one fifth. It has been a proven and successful strategy to inhibit the viral proteases for the treatment of viral infections such as in the cases of human immunodeficiency virus (HIV) and hepatitis $\mathrm{C}$ virus (HCV) and hence screening of protease inhibitors could be a useful approach against COVID-19 Mpro [30]

To investigate the binding mode of Dexamethasone within the SARS-COV2 Mpro computational modelling approach was used. To better comprehend we have investigated the binding interaction of Dexamethasone in comparison with the binding interaction of Remdesivir within the SARS-COV-2 Mpro. Detailed of best free binding energies obtained from best identified docking software are shown in Table. 3

Table 3: Free Binding Energies of Dexamethasone and Remdesivir calculate from CDOCKER and Free Binding Energy in Discovery Studio Client

\begin{tabular}{|c|c|c|}
\hline Compound & Structure & $\begin{array}{c}\text { Free Binding Energy } \\
\qquad \mathbf{G}(\mathrm{kcal} / \mathrm{mol})\end{array}$ \\
\hline Dexamethasone & & -38.7331 \\
\hline Remdesivir & & -33.3358 \\
\hline
\end{tabular}


The binding energy values of Dexamethasone against SARS-COV-2 Mpro were found better than the previously reported antiviral drug Remdesivir. Dexamethasone was able to bind to the new coronavirus SARS-COV-2 Mpro tightly and hence may cause inhibition of SARCOV-2 Mpro.

To investigate the possible reasons for the differences in the binding energies, we also examined the binding interaction of both Dexamethasone and Remdesivir in complexed with SARS-COV-2 Mpro with the help of Discovery studio visualizer. Large number of binding interactions gives the Dexamethasone- SAR-COV-2 Mpro complexes better stability with the binding energy of $-38.7331 \mathrm{kcal} / \mathrm{mol}$. While Remdesivir-Mpro complexes has binding energy of $-33.3358 \mathrm{kcal} / \mathrm{mol}$ which decipher relatively less stability than the Dexamethasone-SARCOV-2 Mpro complex.

For Dexamethasone the binding interactions established upon docking with SAR-COV-2 Mpro are shown in figure 5 and table 4. Detailed analysis of binding interaction between dexamethasone and SARS_COV-2 Mpro Complex revelated that $6 \mathrm{H}$-bonds were formed with the amino acid of SARS-CoV-2 Mpro residues compared to only 3H_bonds formed between Remdesivir and SARS-COV-2 Mpro Complex. Amino acid residue of GLY143, HIS163, GLU166, GLU166, GLN192 and LEU141 of SAR-COV-2 Mpro were involved in making Hbond with Dexamethasone. While in case of Remdesivir- SAR-COV-2 Mpro complex, HIS163, GLU166 and PHE140 were only involved in making H-Bond with Remdesivir. Other type of Binding interaction were also found and summarized in table 4

Table 4: Detailed Binding interactions of Dexamethasone and Remdesivir with Amino acid residue of SAR-

COV-2 Mpro

\begin{tabular}{|l|l|l|}
\hline \multicolumn{2}{|c|}{ Type of Bond } & \multicolumn{1}{c|}{ From } \\
\hline \multicolumn{2}{|c|}{ DEXAMETHASONE } \\
\hline Conventional Hydrogen Bond & A:GLY143:HN & Dexa:O4 \\
\hline Conventional Hydrogen Bond & A:HIS163:HE2 & Dexa:O5 \\
\hline $\begin{array}{l}\text { Conventional Hydrogen Bond;Halogen } \\
\text { (Fluorine) }\end{array}$ & A:GLU166:HN & Dexa:F1 \\
\hline Conventional Hydrogen Bond & A:GLU166:HN & Dexa:O2 \\
\hline Conventional Hydrogen Bond & A:GLN192:HE21 & Dexa:O6 \\
\hline Conventional Hydrogen Bond & Dexa:H57 & A:LEU141:O \\
\hline Carbon Hydrogen Bond & A:ASN142:HA & Dexa:O4 \\
\hline Carbon Hydrogen Bond & A:MET165:HA & Dexa:O2 \\
\hline Halogen (Fluorine) & A:GLU166:O & Dexa:F1 \\
\hline Alkyl & Dexa:C23 & A:MET49 \\
\hline \multicolumn{1}{|c|}{ Remdesivir } & \\
\hline Conventional Hydrogen Bond & A:HIS163:HE2 & :Remdesivir:N14 \\
\hline Conventional Hydrogen Bond & $:$ Remdesivir:H52 & A:GLU166:O \\
\hline Conventional Hydrogen Bond & :Remdesivir:H62 & A:PHE140:O \\
\hline Carbon Hydrogen Bond & A:HIS41:HE1 & :Remdesivir:O3 \\
\hline Carbon Hydrogen Bond & A:PRO168:HD2 & :Remdesivir:O9 \\
\hline Carbon Hydrogen Bond & A:GLN189:HA & :Remdesivir:O6 \\
\hline Carbon Hydrogen Bond & $:$ Remdesivir:H57 & A:GLU166:O \\
\hline Pi-Sulfur & A:MET49:SD & $:$ Remdesivir \\
\hline Pi-Sulfur & A:CYS145:SG & :Remdesivir \\
\hline Pi-Sulfur & A:CYS145:SG & :Remdesivir \\
\hline Pi-Sulfur & A:MET165:SD & :Remdesivir \\
\hline
\end{tabular}


The Hydrogen bonding network of Dexamethasone and Remdesivir was also consistent with those of the reported interacting amino acid residue binding with co-crystal X77 ligand [22] . Dexamethasone has more number of H-bond than both Remdesivir as well as X77 with SARSCOV-2 Mpro as shown in figure. 5 Additionally amino acid residue of ASN142, MET49, MET165 and GLU166 were also involved in forming other hydrophobic interactions with dexamethasone while in case of Remdesivir, amino acid residue of ASN142, MET49, MET165, HIS41, Leu27 and CyS145 were also involved in forming other hydrophobic interactions with Remdesivir. From the overall molecular modelling analysis, the identified set of binding interaction formed by Dexamethasone can be considered as contributing factor, even though we agree that no experimentally supported inhibition and binding assay was conducted.
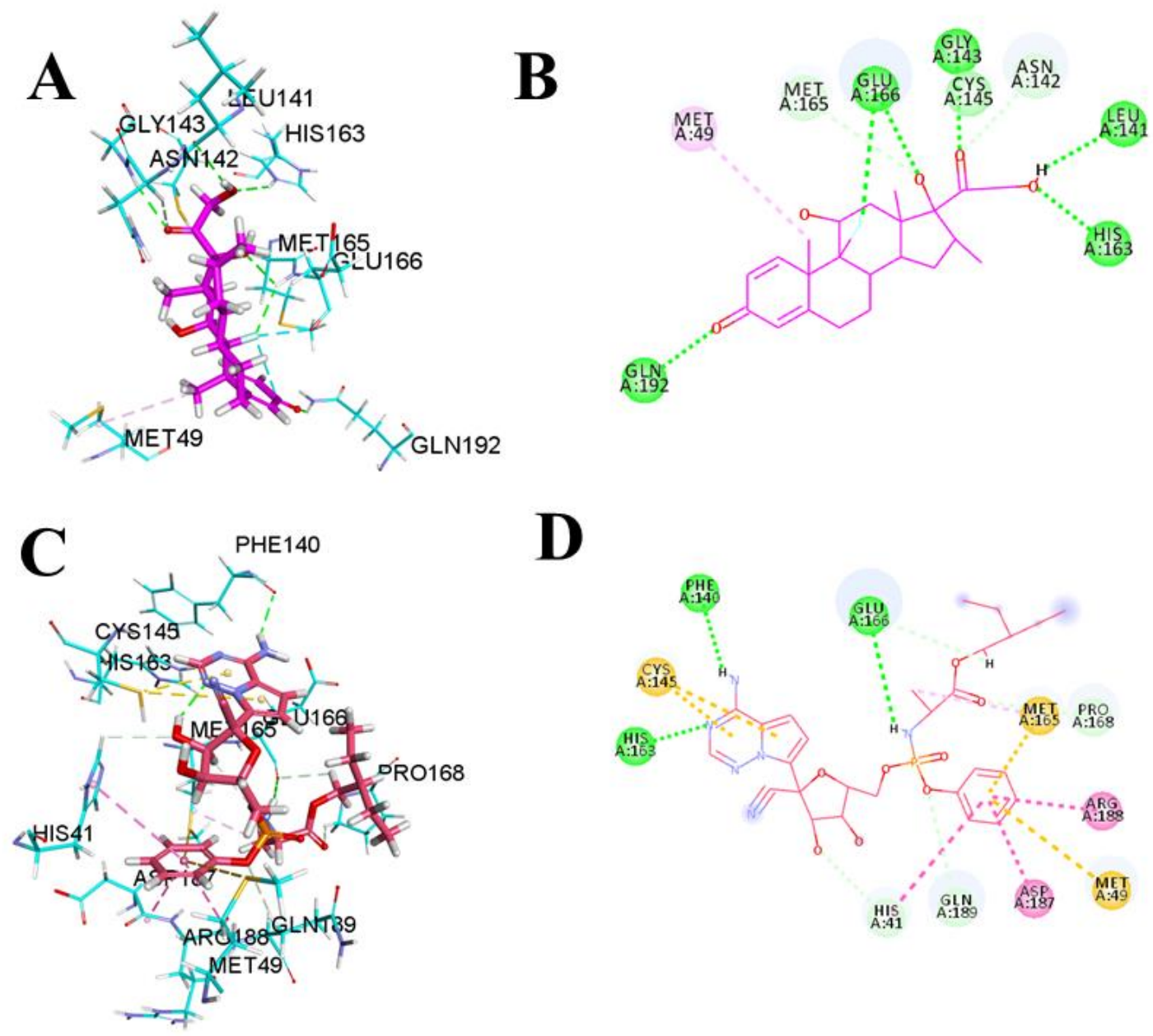

Figure 5: Lowest energy binding conformations of Dexamethasone and Remdesivir bound to SARS-CoV-2 Mpro generated by molecular docking. A and $\mathbf{B}$ represent 3D and 2D representation of Dexamethasone (pink colour) which was well stabilised by various amino acid residue (cyan colour) within the active pocket of SARS-CoV2 Main Protease . C and D represent 3D and 2D representation of Remdesivir (orange colour) which was well stabilised by various amino acid residue (cyan colour) within the active pocket of SARS-CoV-2 Mpro Hydrogen bonding are shown in green dotted line, pi-sulfur are shown in golden dotted line while pi-pi T-Shaped interaction are shown in pink dotted line

\section{Conclusion}

On 30 January 2020, the WHO declared the COVID-19 pandemic as a Public Health Emergency of International Concern (PHEIC). The newly emerged SAR-COV-2 is a 
worldwide health concern which is around 8, 061, 550 cases and caused death of 44, 290 till 17 June 2020. On 17 Jan 2020, WHO applauds the initial clinical trial results from the United Kingdom that show dexamethasone, a corticosteroid, can be lifesaving for patients who are critically ill with COVID-19. In the present study we have attempted to investigate blinding interaction of Dexamethasone with SARS-COV-2 Mpro in comparison with the previously reported Anti4viral drug Remdesivir against SARS-COV-2 Mpro We suggest that dexamethasone has excellent results for binding with SAR-COV-2 Mpro than the Remdesivir. The higher binding of dexamethasone is own to establishing grater number of hydrogen bond than the Remdesivir antiviral drug. Along with reported Hydrogen bond with co-crystal ligand X77 and Remdesivir, Dexamethasone has three additional hydrogen bond which make the dexamethasone better binder than Remdesivir. Invitro investigation of dexamethasone with SAR-COV-2 Mpro need to be investigated for getting more insight into the inhibitory potential of Dexamethasone against COVID-19.

Acknowledgments: We would like to thank OpenEye Scientific Software, BIOVIA DISCOVERY Studio Client and BIOMOLTECH for providing a free academic and evaluation license for performing in silico studies

\section{Reference}

1. Bogoch, I.I., et al., Pneumonia of Unknown Etiology in Wuhan, China: Potential for International Spread Via Commercial Air Travel. Journal of Travel Medicine, 2020.

2. Organization, W.H., Surveillance case definitions for human infection with novel coronavirus ( $n \mathrm{CoV}$ ): interim guidance v1, January 2020. 2020, World Health Organization.

3. https://www.who.int/emergencies/diseases/novel-coronavirus-2019/situation-reports, Coronavirus disease (COVID-19) Situation Report - 149. 2020.

4. Li, H., et al., Coronavirus disease 2019 (COVID-19): current status and future perspective. International journal of antimicrobial agents, 2020: p. 105951.

5. $\quad$ Li, G. and E. De Clercq, Therapeutic options for the 2019 novel coronavirus (2019$n C o V)$. 2020, Nature Publishing Group.

6. Mittal, L., et al., Identification of potential molecules against COVID-19 main protease through structure-guided virtual screening approach. Journal of Biomolecular Structure and Dynamics, 2020(just-accepted): p. 1-26.

7. Ledford, H., Coronavirus breakthrough: dexamethasone is first drug shown to save lives. 2020.

8. Low-cost dexamethasone reduces death by up to one third in hospitalised patients with severe respiratory complications of COVID-19. http://www.ox.ac.uk/news/2020-0616-low-cost-dexamethasone-reduces-death-one-third-hospitalised-patients-severe, 2020.

9. https://www.who.int/news-room/detail/16-06-2020-who-welcomes-preliminaryresults-about-dexamethasone-use-in-treating-critically-ill-covid-19-patients, $\quad W H O$ welcomes preliminary results about dexamethasone use in treating critically ill COVID-19 patients. 2020.

10. Bunim, J.J., et al., Studies on dexamethasone, a new synthetic steroid, in rheumatoid arthritis-a preliminary report. Arthritis \& Rheumatism: Official Journal of the American College of Rheumatology, 1958. 1(4): p. 313-331.

11. Hollander, J.L., Clinical use of dexamethasone: role in treatment of patients with arthritis. Journal of the American Medical Association, 1960. 172(4): p. 306-310. 
12. Stoll, B.A., Dexamethasone in advanced breast cancer. Cancer, 1960. 13(5): p. 10741080 .

13. Organization, W.H., The selection of essential drugs: report of a WHO expert committee [meeting held in Geneva from 17 to 21 October 1977]. 1977.

14. Villar, J., et al., Rationale for prolonged corticosteroid treatment in the acute respiratory distress syndrome caused by coronavirus disease 2019. Critical Care Explorations, 2020. 2(4).

15. Elfiky, A.A., SARS-CoV-2 RNA dependent RNA polymerase (RdRp) targeting: An in silico perspective. Journal of Biomolecular Structure and Dynamics, 2020: p. 1-9.

16. Elmezayen, A.D., et al., Drug repurposing for coronavirus (COVID-19): in silico screening of known drugs against coronavirus 3CL hydrolase and protease enzymes. Journal of Biomolecular Structure and Dynamics, 2020: p. 1-13.

17. Enayatkhani, M., et al., Reverse vaccinology approach to design a novel multi-epitope vaccine candidate against COVID-19: an in silico study. Journal of Biomolecular Structure and Dynamics, 2020: p. 1-16.

18. Enmozhi, S.K., et al., Andrographolide as a potential inhibitor of SARS-CoV-2 main protease: an in silico approach. Journal of Biomolecular Structure and Dynamics, 2020: p. 1-7.

19. Crosby, J.C., et al., COVID-19: A review of therapeutics under investigation. Journal of the American College of Emergency Physicians Open, 2020.

20. Boopathi, S., A.B. Poma, and P. Kolandaivel, Novel 2019 coronavirus structure, mechanism of action, antiviral drug promises and rule out against its treatment. Journal of Biomolecular Structure and Dynamics, 2020: p. 1-10.

21. Yu, R., et al., Computational screening of antagonist against the SARS-CoV-2 (COVID19) coronavirus by molecular docking. International Journal of Antimicrobial Agents, 2020: p. 106012.

22. Mesecar, A., A taxonomically-driven approach to development of potent, broadspectrum inhibitors of coronavirus main protease including SARS-CoV-2 (COVID-19). Be Publ, 2020.

23. Zhavoronkov, A., et al., Potential non-covalent SARS-CoV-2 3C-like protease inhibitors designed using generative deep learning approaches and reviewed by human medicinal chemist in virtual reality. 2020.

24. Pilot, P., Dassault Systèmes BIOVIA, discovery studio modelling environment. 2020, Release.

25. Ellingson, B.A., et al., Efficient calculation of SAMPLA hydration free energies using $O M E G A$, SZYBKI, QUACPAC, and Zap TK. Journal of computer-aided molecular design, 2014. 28(3): p. 289-298.

26. McGann, M., FRED pose prediction and virtual screening accuracy. Journal of chemical information and modeling, 2011. 51(3): p. 578-596.

27. McGann, M., FRED and HYBRID docking performance on standardized datasets. Journal of computer-aided molecular design, 2012. 26(8): p. 897-906.

28. Stroganov, O.V., et al., Lead finder: an approach to improve accuracy of proteinligand docking, binding energy estimation, and virtual screening. Journal of Chemical Information and Modeling, 2008. 48(12): p. 2371-2385.

29. Grein, J., et al., Compassionate use of remdesivir for patients with severe Covid-19. New England Journal of Medicine, 2020. 382(24): p. 2327-2336.

30. Ghosh, A.K., H.L. Osswald, and G. Prato, Recent Progress in the Development of HIV1 Protease Inhibitors for the Treatment of HIV/AIDS. Journal of medicinal chemistry, 2016. 59(11): p. 5172-5208. 
